\title{
Cervical tracheal resection with cricotracheal anastomosis: experience in adults with grade III-IV tracheal stenosis
}

\author{
A M A EL-FATTAH ${ }^{1}$, E KAMAL $^{1}, \mathrm{H} \mathrm{E} \mathrm{AMER}^{1}, \mathrm{M} \mathrm{FOUDA}^{2}$, A E M A ELWAHAB ${ }^{1}$, \\ A TAWFIK ${ }^{1}$ \\ ${ }^{1}$ Otolaryngology Department, and ${ }^{2}$ Cardiothoracic Surgery Department, Faculty of Medicine, Mansoura \\ University, Egypt
}

\begin{abstract}
Introduction: Laryngotracheal stenosis is currently one of the most common complications associated with nasal and orotracheal intubation and tracheotomy. Once established, tracheal stenosis can be a complex and difficult problem to manage.

Patients and methods: We retrospectively analysed 2004-2010 data for 12 male patients with postintubation cervical tracheal stenosis (grade III-IV) treated in the otolaryngology department, Mansoura University Hospitals. All patients had a tracheostomy at presentation, and all underwent tracheal resection with primary cricotracheal anastomosis and suprahyoid release.

Results: Grade III stenosis was present in five patients (41.7 per cent) and grade IV stenosis in seven patients (58.3 per cent). The length of trachea resected ranged from 2 to $4 \mathrm{~cm}$, representing one to four tracheal rings. In all 12 patients, the procedure allowed successful tracheotomy decannulation. Minor complications comprised surgical emphysema $(n=2)$ and wound infection $(n=1)$, and were managed conservatively. Major complications consisted of restenosis $(n=3)$, managed in two patients by repeated dilatation; one patient was lost to follow up.

Conclusion: Segmental tracheal resection with cricotracheal anastomosis was successful in 11/12 (92 per cent) patients with severe cervical tracheal stenosis. The strategy for treatment of airway stenosis is now well established and success rates are high, with minimal or no sequelae.
\end{abstract}

Key words: Tracheal Stenosis; Surgery; Endotracheal Intubation; Complications

\section{Introduction}

Over the last few decades, laryngotracheal stenosis has become recognised as a pathological otorhinolaryngological condition. ${ }^{1}$ The increased use of tracheotomy and nasal and orotracheal intubation (e.g. in intensive care units) may explain the increasing prevalence of laryngotracheal stenosis, with recently reported incidences ranging from 0.6 to 21 per cent. Advances in critical care medicine have been thought to contribute to an increase in the general incidence of tracheal injuries, due to improved survival following intensive care admission. Laryngotracheal stenosis is currently considered one of the most common complications associated with such intervention. $^{2-5}$

Once established, tracheal stenosis can be a complex and difficult problem to manage. Patient comorbidity, the degree and length of stenosis, and the propensity for restenosis need to be considered when determining the best treatment option. Several treatment modalities have been used, ranging from less invasive procedures, such as sequential airway dilatation, laser treatment and stent placement, to major airway surgical resection and reconstruction. ${ }^{6-8}$ Although dilatation and laser treatment are safe, well tolerated procedures, they usually result in only temporary improvement of symptoms; recurrence is frequent, requiring repeated procedures without a definitive result. Tracheal resection is well established as the definitive treatment for benign tracheal stenosis and has been shown to yield excellent results in selected patients. ${ }^{3}$

Early in the twentieth century, it was believed that only $2 \mathrm{~cm}$ of trachea could be safely resected and reconstructed. This belief was later challenged as a result of tracheal anatomy studies and the advent of tracheal mobilisation and releasing manoeuvres. Grillo and Colleagues, a major contributor to this clinical arena for decades, found that the average length of trachea that could be resected and primarily anastomosed was $6.4 \mathrm{~cm}$, or up to 58 per cent of the total tracheal length when cervical flexion and releasing 
manoeuvres were used. ${ }^{9,10}$ Laccourreye et al. reported that tracheal segments of up to $3.5 \mathrm{~cm}$ could be safely resected and reconstructed without the use of releasing manoeuvres. ${ }^{11}$ However, other authors reported that muscle-releasing techniques were required for the successful management of tracheal stenosis of more than $2 \mathrm{~cm}^{12}$

Releasing manoeuvres have proven successful in reducing anastomotic tension. Therefore, some surgeons have advocated the routine use of such manoeuvres when performing tracheal resection and primary anastomosis. ${ }^{13-15}$ The theoretical advantage of decreased anastomotic tension is a reduced incidence of wound complications such as granulation, poor healing and restenosis. The disadvantages of releasing manoeuvres include an increased incidence of post-operative swallowing difficulties, particularly following infrahyoid release. In one major series, laryngeal release was associated with a higher incidence of aspiration and laryngeal dysfunction, ranging from 20 per cent in patients undergoing suprahyoid release to 44 per cent in those managed with thyrohyoid release. $^{3}$ In a smaller series reported by Biller and Munier, all patients underwent combined infrahyoid and inferior constrictor release; two of the 10 patients required prolonged nasogastric feeding secondary to dysphagia, a complication otherwise rarely reported in the tracheal resection literature. ${ }^{12}$

The purpose of the present study was to review the experience of the Mansoura University Hospitals otolaryngology department, in cooperation with the cardiothoracic surgery department, in applying singlestaged tracheal resection with cricotracheal anastomosis in patients with severe cervical tracheal stenosis (i.e. grade III or IV), and to compare the outcomes to those reported in previously published case series.

\section{Patients and methods}

The approval of the otolaryngology department research and ethics committee was obtained prior to study commencement.

We conducted a retrospective chart review of patients with tracheal stenosis who were admitted to the Mansoura University Hospitals otolaryngology department between January 2004 and January 2010. The review identified patients who had undergone tracheal resection with primary cricotracheal anastomosis performed by the authors over the six-year study period. All patients were managed with suprahyoid releasing manoeuvres.

Data were obtained from each patient's medical chart, including: age at time of surgery; comorbidity; length and degree of stenosis; post-operative complications; and any required interventions or additional airway surgery.

Pre-operative assessment included careful laryngotracheal endoscopic examination to determine the degree of stenosis, to verify the grade of inflammation and oedema of the subglottis and trachea, to determine the integrity of the vocal folds, and to measure the extent of laryngotracheal involvement. Computed tomography (CT) of the neck and chest was conducted to determine the extent of stenosis and the remaining linear amount of normal airway, and to assess the extraluminal region. The Meyer and Cotton grading system was used to categorise the clinical severity of patients' stenoses (grade III $=70-99$ per cent stenosis; grade IV = no detectable lumen), and to enable comparison with other reported series. ${ }^{16}$

The length of tracheal stenosis was estimated at the time of the surgery. When tracheal rings or their remnants were readily countable, this number was noted. When fibrosis and scarring precluded the counting of rings, the length of stenosis was measured in centimetres and noted in the operative report. We also noted the time of resumption of oral intake, any complaints of dysphagia and any post-operative aspiration.

Each patient's individual management protocol was determined at a group meeting including the otolaryngological and cardiothoracic teams. Explanations of treatment options were regularly offered to all patients and discussed with them, in order to clarify the advantages and side effects of the different treatment regimens used. An informed consent form, signed by the patient or their guardian, was always obtained before surgery.

\section{Surgical technique}

The surgical approach for a segmental airway resection with primary anastomosis was performed according to the technique described by Pearson et al. and Grillo. ${ }^{17,18}$ All patients were already tracheostomised at the time of admission because of severe airway compromise. Anaesthesia was maintained via an endotracheal tube placed through the tracheostomy below the stenosis. Immediately before the external approach, the stenotic segment was again endoscopically identified and evaluated.

The operative approach was via a cervical collar incision; there was no need for sternal splitting or median sternotomy in any patient. Patients were initially placed in cervical extension using a shoulder roll, which was removed when tracheal anastomosis was performed. The stenotic airway was dissected from the surrounding scar tissue, and circumferential tracheal resection was performed together with resection of the tracheostomy stoma. The recurrent laryngeal nerve was not identified in any patient; injury to this structure was minimised by meticulous dissection of the area immediately adjacent to the tracheal surface. Throughout surgery, including the actual anastomosis, ventilation was performed via a cross-field endotracheal tube placed in the distal trachea.

Previous reports have described a number of releasing manoeuvres to enable a tension-free anastomosis. In our series, we used the suprahyoid release technique of Montgomery in all patients. ${ }^{19}$ In comparison with other releasing manoeuvres, this technique is well tolerated by the patient and easy to accomplish, and 
involves releasing the larynx or hyoid bone from its upper muscular attachments. Interrupted 2-0 Prolene sutures were used for repair of both the cartilaginous trachea and the membranous trachea. After completing the insertion of sutures in the membranous trachea and the lateral walls, the anaesthetist was asked to advance the endotracheal tube in the usual way transorally across the anastomotic site, and the rest of the anastomosis was completed.

In our last two cases, fibrin glue was applied at the site of anastomosis in order to seal the anastomotic site from any air leakage, enhancing healing and consequently reducing inflammation and fibrosis.

A passive drain was placed deep in the wound.

None of our patients was placed in cervical flexion using a chin-to-sternal suture, but rather were fitted with a reversed hard neck collar to keep their neck flexed.

Most of our patients (eight of 12) were extubated at the end of the operation, within the operating theatre. The remaining four patients were extubated in the first 24 hours post-operatively, due to the surgeon's preference to keep the patient orotracheally intubated overnight in order to minimise air leakage from the anastomotic site. All four patients were kept under observation for 24 hours in the intensive care unit. These patients were extubated the following morning and allowed to eat the same day; diet was advanced as tolerated.

To reduce the risk of laryngeal oedema, post-extubation management included elevating the head of the bed, limiting voice usage, and administering aerosolised and/or systemic corticosteroids and antibiotics. The drain was left in place for three days. Post-operative care included airway observation, pulmonary toilet, and the provision of humidified air or oxygen in order to minimise crusting at the anastomosis as well as to reduce mucus plugging.

Follow up included clinical evaluation and endoscopy at one, three and six months post-operatively and when indicated. The long-term outcome was classified as either excellent (i.e. no or only minor complications), satisfactory (i.e. major complications but successful decannulation from the tracheostomy) or unsatisfactory (i.e. decannulation unsuccessful). The follow-up period ranged from six to 12 months (median, 6.5 months).

\section{Results}

From January 2004 to January 2010, 12 patients with severe upper tracheal stenosis (i.e. grade III-IV) were identified and treated at the Mansoura University Hospitals otolaryngology department. These 12 patients underwent tracheal resection and primary cricotracheal anastomosis, using the suprahyoid releasing manoeuvre as the standard technique to obtain a tension-free anastomosis. Table I summarises the 12 patients' data. The patients' ages ranged from 22 to 46 years (median, 32.7). All 12 patients were male. The aetiology of tracheal stenosis was postintubation in all cases, following intensive care unit admission due to either multiple trauma $(n=4)$, head trauma $(n=4)$, or cardiac $(n=1)$ or neurological $(n=3)$ causes. All patients had undergone tracheostomy on presentation (to either the Emergency Mansoura University Hospital or another hospital), due to significant airway compromise caused by stenosis. One patient had previously undergone unsuccessful laser surgery in another institution before being referred to our department.

Use of the adapted Meyer and Cotton grading system allowed methodical classification of the degree of tracheal stenosis. Our patients' stenoses were graded as follows: grade III, 41.7 per cent (five patients); and grade IV, 58.3 per cent (seven patients). The length of trachea resected ranged from 2 to $4 \mathrm{~cm}$, representing one to four tracheal rings.

All 12 patients had a tracheostomy in place at the time of tracheal resection, and were successfully

\begin{tabular}{|c|c|c|c|c|c|c|c|c|}
\hline \multicolumn{9}{|c|}{$\begin{array}{r}\text { TABLE I } \\
\text { UPPER TRACHEAL RESECTION WITH PRIMARY CRICOTRAC }\end{array}$} \\
\hline Pt no & Age (y) & $\begin{array}{l}\text { Tracheotomy } \\
\text { at surgery? }\end{array}$ & $\begin{array}{l}\text { Stenosis } \\
\text { grade }\end{array}$ & $\begin{array}{l}\text { Trachea length } \\
\text { resected }\end{array}$ & Complications & Interventions & Extubated? & Outcome \\
\hline 1 & 46 & Yes & IV & 3 rings & Partial stenosis & $\begin{array}{l}\text { Repeated dilatation, } \\
\text { tracheostomy }\end{array}$ & Yes & Satisfactory \\
\hline 2 & 34 & Yes & IV & $2.5 \mathrm{~cm}$ & $\begin{array}{l}\text { Surgical } \\
\text { emphysema }\end{array}$ & Conservative & Yes & Satisfactory \\
\hline 3 & 39 & Yes & III & $3 \mathrm{~cm}$ & - & - & Yes & Excellent \\
\hline 4 & 29 & Yes & IV & 2 rings & Wound infection & Antibiotics & Yes & Satisfactory \\
\hline 5 & 31 & Yes & III & $3.5 \mathrm{~cm}$ & - & - & Yes & Excellent \\
\hline 6 & 42 & Yes & III & 2 rings & - & - & Yes & Excellent \\
\hline 7 & 24 & Yes & IV & 4 rings & Partial stenosis & Repeated dilatation & Yes & Satisfactory \\
\hline 8 & 33 & Yes & III & $2.5 \mathrm{~cm}$ & - & - & Yes & Excellent \\
\hline 9 & 25 & Yes & IV & $4 \mathrm{~cm}$ & Restenosis & Lost to follow up & Unknown & $\begin{array}{l}\text { Missing } \\
\text { data }\end{array}$ \\
\hline 10 & 22 & Yes & IV & $3 \mathrm{~cm}$ & $\begin{array}{l}\text { Surgical } \\
\text { emphysema }\end{array}$ & Conservative & Yes & Satisfactory \\
\hline 11 & 40 & Yes & IV & 3 rings & - & - & Yes & Excellent \\
\hline 12 & 27 & Yes & III & 3 rings & - & - & Yes & Excellent \\
\hline
\end{tabular}

Pt no $=$ patient number; $y=$ years 
decannulated at the end of the procedure. Eight patients (66.7 per cent) were successfully extubated at the completion of surgery; the remaining four (33.3 per cent) were extubated on post-operative day one.

No nasogastric or other feeding tubes were used in any of the patients. All patients started oral feeding on post-operative day one.

Only temporary, insignificant dysphagia and aspiration were noted for the 12 patients. There were no cases of post-operative pneumonia or recurrent laryngeal nerve injury.

Two patients (16.7 per cent) developed surgical emphysema of the neck on post-operative days four and five, variously, but no airway compromise was noted. One patient ( 8.3 per cent) had a wound infection on post-operative day seven. All three patients were managed conservatively, with no further sequelae.

The most frequent major complication was restenosis at the site of anastomosis, which occurred in three patients ( 25 per cent) and required a tracheostomy in one. In two of these patients, repeated dilatation was successful and no further surgical intervention was performed. The third patient was lost to follow up and his data were thus incomplete.

The patients' follow-up duration ranged from six to 12 months. Eleven of the 12 were relieved of their airway obstruction as a result of surgery (92 per cent).

Fibrin glue was used at the site of anastomosis in two cases, with the aim of avoiding the minor complication of partial anastomotic breakdown (observed in the two patients with surgical emphysema) and the major complication of restenosis (observed in three patients); these goals were successfully achieved.

The outcome was considered excellent in nine patients and satisfactory in two patients; one patient was lost to follow up. No patients were observed to have an unsatisfactory outcome. However, outcome classification was based on clinical evaluation rather than objective investigation (e.g. with flow volume loops or bronchoscopy). A thorough study measuring objective outcome parameters may help determine the quantitative success of airway anastomosis in this patient group.

\section{Discussion}

In the present study of 12 patients undergoing tracheal resection with primary anastomosis, 92 per cent were successfully extubated.

It is generally difficult to compare surgical outcomes across institutions, surgeons and eras. However, tracheal resection with primary anastomosis is quite a standardised procedure and has not changed significantly since the first major series were reported. This enables meaningful comparison between papers.

Despite improved patient care, laryngotracheal stenosis remains an important iatrogenic complication of tracheal intubation. The diagnosis and treatment of laryngotracheal stenosis is a challenge requiring a multidisciplinary approach, which may involve otorhinolaryngologists, thoracic surgeons and intensive care clinicians. ${ }^{2-4}$ Severe tracheal stenosis (i.e. grade III-IV) represents a major therapeutic challenge. Such patients have a critically compromised airway and tracheostomy is essential, especially for cases with a stenosis longer than $1 \mathrm{~cm}$, a circumferential stenosis or no residual cartilaginous support. Pre-operative assessment must define the precise topography of the injury (including the exact location and length of stenosis), the length of tracheal involvement, and the presence of inflammation and oedema at the stenosis border. Patients with severe inflammatory signs should be excluded from surgery and re-evaluated after a suitable period of observation, to determine whether the stenosis has stabilised.

Rigid endoscopy represents the best pre-operative diagnostic procedure for patients with inflammatory tracheal stenosis. Helical (spiral) CT with a 1-1.5 thin section allows high quality multiplanar reconstruction, permitting detailed evaluation of tracheal stenosis. ${ }^{20}$ We believe that $\mathrm{CT}$ scanning always adds further information to that derived from rigid endoscopy, in cases in which the lumen is too stenosed to be traversed and the exact length of involved trachea thus cannot be measured, and especially in patients who have undergone a previous surgical tracheal procedure. Computed tomography scanning can also obtain more information on the extraluminal region.

Complete removal of the stenotic segment, preservation of vascularisation and innervation, and careful submucosal suturing with minimum tension are of paramount importance for surgical success. ${ }^{21,22}$ To achieve this last goal, many laryngotracheal releasing manoeuvres have been proposed, such as tracheal dissection, suprahyoid liberation and even dissection from the anterior mediastinum. ${ }^{13-15}$ However, in Merati and colleagues' series of 17 tracheal segmental resections with primary anastomosis, with a success rate of 94 per cent, adjuvant releasing manoeuvres were not performed in order to avoid the swallowing problems associated with such manoeuvres. ${ }^{23}$ In our series, the above-mentioned tenets of surgical success were respected, and the suprahyoid releasing manoeuvre was standardised in all patients. Early extubation was achieved in all 12 patients. This contrasts with the delayed extubation reported in other published series, in patients with a stenosis located in the upper trachea and involving the cricoid ring, requiring thyrotracheal or cricotracheal anastomosis., ${ }^{31,24}$ Only insignificant and temporary dysphagia or aspiration were reported for our 12 patients, despite use of the suprahyoid releasing manoeuvre. We believe that this manoeuvre is central to achieving good results and early extubation, and also avoids the need for the disturbing practice of chin-to-chest suturing.

In the current series, interrupted, non-absorbable 2-0 sutures were used for anastomosis in all patients, and no major complications were observed related to anastomotic granulation formation. The most common complication of this surgical procedure seems to be 
partial restenosis, which occurred in three patients in our series, and surgical emphysema due to partial anastomosis dehiscence, which occurred in two patients. However, repeated dilatation was successful in two of the three former cases, with no further surgical intervention required, and conservative management was sufficient in the latter two cases. The use of fibrin glue at the anastomotic site has been suggested to avoid such problems; this was successfully used in our last two patients, but more thorough study in larger series is required.

- This study examined 12 patients with postintubation severe cervical tracheal stenosis, treated over a six-year period

- Five patients (41.7 per cent) had grade III stenosis and seven patients (58.3 per cent) had grade IV stenosis

- All patients underwent segmental tracheal resection with primary cricotracheal anastomosis and suprahyoid release, removing 2-4 cm of trachea (one to four tracheal rings)

- All patients were successfully decannulated post-operatively

- Minor complications comprised surgical emphysema $(n=2)$ and wound infection $(n=1)$

- Three patients restenosed; two were managed by repeated dilatation and one was lost to follow up

- A successful outcome was achieved in $11 / 12$ ( 92 per cent) patients

Our results confirm that surgery represents the best treatment for benign severe tracheal stenosis (i.e. grade III-IV). Our findings also indicate that the suprahyoid releasing manoeuvre can be used to obtain tension-free anastomosis, without the previously reported major swallowing problems. There is no need to place a chin-to-chest suture, as fitting the patient with a reversed hard neck collar is adequate to avoid sudden neck extension and consequent rupture of the anastomosis. The additional use of fibrin glue at the anastomosis site should be considered, to enhance healing. Careful pre-operative assessment and preparation, together with meticulous surgical technique, are mandatory to obtain good results.

\section{Conclusion}

Laryngotracheal stenosis remains one of the most common and serious complications of endotracheal intubation. Currently, the management procedure of choice for severe upper tracheal stenosis comprises resection of the stenotic segment with primary cricotracheal anastomosis. Basic releasing manoeuvres, such as suprahyoid release, allow a good length of trachea to be resected without complication, and have encouraging results and low morbidity. In the present series, a 92 per cent success rate was achieved.

\section{References}

1 Primov-Fever A, Talmi YP, Yellin A, Wolf M. Cricotracheal resection for airway reconstruction: the Sheba Medical Center experience. Isr Med J 2006;8:543-8

2 Pearson FG, Andrews MJ. Detection and management of tracheal stenosis following cuffed tube tracheostomy. Ann Thorac Surg 1971;12:359-74

3 Grillo HC, Donahue DM, Mathisen DJ, Wain JC, Wright CD. Postintubation tracheal stenosis: treatment and results. J Thorac Cardiovasc Surg 1995;109:486-93

4 Stauffer JL, Olson DE, Petty TL. Complications and consequences of endotracheal intubation and tracheostomy: a prospective study in 150 critically ill adult patients. Am J Med 1981;70: $65-76$

5 Softah A. Rigid bronchoscopic dilatation of postintubation tracheal stenosis. West Afr J Med 2005;24:234-8

6 Ciccone AM, De Giacomo T, Venuta F, Ibrahim M, Diso D, Coloni GF et al. Operative and non-operative treatment of benign subglottic laryngotracheal stenosis. Eur J Cardiothorac Surg 2004:26:818-22

7 Macchiarini P, Chapelier A, Lenot B, Cerrina J, Dartevelle P. Laryngotracheal resection and reconstruction for post-intubation subglottic stenosis. Eur J Cardiothorac Surg 1993;7:300-5

8 Pearson FG, Brito-Filomeno L, Cooper JD. Experience with partial cricoid resection and thyrotracheal anastomosis. Ann Otol Rhinol Laryngol 1986;95:582-5

9 Grillo HC, Mathisen DJ, Ashiku SK, Wright CD, Wain JC. Successful treatment of idiopathic laryngotracheal stenosis by resection and primary anastomosis. Ann Otol Rhinol Laryngol 2003;112:798-800

10 Heitmiller RF. Tracheal release maneuvers. Chest Surg Clin N Am 2003;13:201-10

11 Laccourreye O, Naudo P, Brasnu D, Jouffre V, Cauchois R, Laccourreye $\mathrm{H}$. Tracheal resection with end-to-end anastomosis for isolated postintubation cervical trachea stenosis: long-term results. Ann Otol Rhinol Laryngol 1996;105:944-8

12 Biller HG, Munier MA. Combined infrahyoid and inferior constrictor muscle release for tension-free anastomosis during primary tracheal repair. Otolaryngol Head Neck Surg 1992; 107:430-3

13 Dedo HH, Fishman NH. Laryngeal release and sleeve resection for tracheal stenosis. Ann Otol Rhinol Laryngol 1969;78: 286-96

14 Zitsch RP, Mullins B, Temper J, Davis WE. Suprahyoid and inferior constrictor release for laryngeal lowering. Arch Otolaryngol 1995;121:1310-13

15 Dedo HH, Fishman NH. The results of laryngeal release, tracheal mobilization and resection for tracheal stenosis in 19 patients. Laryngoscope 1973;83:1204-10

16 Myer CM, O'Connor DM, Cotton RT. Proposed grading system for subglottic stenosis based on endotracheal tube sizes. Ann Otol Rhinol Laryngol 1994;103:319-23

17 Pearson FG, Cooper JD, Nelems JM, Van Nostrand AWP Primary tracheal anastomosis after resection of the cricoid cartilage with preservation of recurrent laryngeal nerves. $J$ Thorac Cardiovasc Surg 1975;70:806-16

18 Grillo HC. The management of tracheal stenosis following assisted respiration. J Thorac Cardiovasc Surg 1969:57:52-71

19 Montgomery W. Suprahyoid release for tracheal anastomosis. Arch Otolaryngol 1974;99:255-60

20 Quint LE, Whyte RI, Kazerooni EA, Martinez FJ, Cascade PN, Lynch JP et al. Stenosis of the central airways: evaluation by using helical CT with multiplanar reconstructions. Radiology 1995;194:871-7

21 Wynn R, Har-El G, Lim JW. Tracheal resection with end-to-end anastomosis for benign tracheal stenosis. Ann Otol Rhinol Laryngol 2004;113:613-17

22 Wolf M, Shapira Y, Talmi YP, Novikov I, Kronenberg J, Yellin A. Laryngo-tracheal anastomosis: primary and revised procedures. Laryngoscope 2001;111:622-7 
23 Merati AL, Rieder AA, Patel N, Park DL, Girod D. Does successful segmental tracheal resection require releasing maneuvers? Otolaryngol Head Neck Surg 2005;133:372-6

24 Marques P, Leal L, Spratley J, Cardoso E, Santos M. Tracheal resection with primary anastomosis: 10 years experience. Am J Otolaryngol 2009;30:415-18

Address for correspondence:

Dr Ahmed Musaad Abd El-Fattah,

Otolaryngology Department,
Faculty of Medicine, Mansoura University,

Mansoura, Egypt

Fax: +2 0502267016

E-mail: ahmusaad@mans.edu.eg

Dr A M A El-Fattah takes responsibility for the integrity of the content of the paper

Competing interests: None declared 